\title{
Efficacy and safety of a Ginkgo biloba extract
}

\author{
PL Le Bars ${ }^{1,2, *}$ and J Kastelan ${ }^{2,3}$ \\ ${ }^{1}$ New York University Medical Center, New York, USA: ${ }^{2}$ Memory Centers of America, 225 East 64th Street, \\ Suite C-102,10021 New York, USA: ${ }^{3} \mathrm{HZI}$ Research Center, Elmsford, NY, USA
}

\begin{abstract}
This review of the literature documents the efficacy of a standard extract of Ginkgo biloba (EGb) in managing signs and symptoms associated with memory disorders and dementia. Analysis of the discrepant findings reveals that study outcomes may vary with the type of population studied, the outcome measurements selected, and the dosing tested. Overall, the efficacy of EGb was more frequently reported in trials enrolling dementia patients than healthy volunteers. In contrast to narrow memory tests, broad cognitive assessments were more likely to detect the treatment effect. Although a dose-response relationship is not yet established, $240 \mathrm{mg}^{\text {day }^{-1} \mathrm{EGb}}$ seems to show a higher rate of treatment response than does $120 \mathrm{mg}^{\mathrm{day}^{-1}}$. Regarding safety, in all trials reviewed the adverse event profile of EGb was not different from that of the placebo.
\end{abstract}

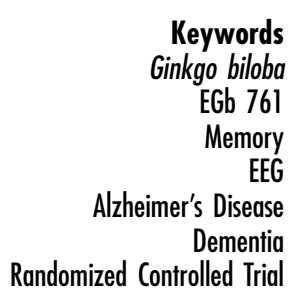

The last 20 years have produced a large body of evidence demonstrating the efficacy of Ginkgo biloba in the management of symptoms of a range of disorders affecting the nervous and vascular systems. However, the very same fields that demonstrated efficacy have simultaneously yielded contradictory results. While an underpowered study or a study applying an inadequate design could occasionally explain a negative outcome, many trials meeting standard criteria nevertheless led to inconsistent findings. An analysis of the discrepant results reveals at least three confounding factors: (1) the characteristics of the study population, (2) the type of outcome measurements, and (3) the ginkgo dosing tested in the trial.

Each of these factors could be a source of discrepancy among studies and may be an important element to be considered for ensuring success in the use of the herbal remedy. This review will summarize the results of studies testing the effects of a standardized extract of ginkgo on cognitive domains.

\section{Extract of ginkgo and standardization}

Standardization of herbal remedies in general and of ginkgo in particular is an essential factor to reduce variability in research programmes. Most of the studies reviewed below tested a standardized extract of Ginkgo biloba, EGb $761^{\circledR}$ (EGb), whose formulation and composition is identical to the product registered in Germany under the name Tebonine ${ }^{\circledR}$ forte (Dr. Willmar Schwabe Pharmaceuticals, Karlsruhe). A standardized method is used to prepare the concentrated extract exclusively from the dried leaves of the ginkgo tree and ensures stability in the ratio of the most active constituents, ginkgo-flavonoids (24\%) and terpene-lactones (6\%). The standardization also aims to reduce the concentration of ginkgolic acids (i.e. less than $5 \mathrm{ppm}$ ) that are considered allergenic. Seventy percent of the total constituents of the extract are systematically verified to ensure EGb's consistent quality, regardless of the origin of the raw material.

\section{Memory and neuropsychological testing}

Neuropsychological testing of memory and related cognitive processes provides a favourable domain in which to demonstrate the influence of two confounding factors on the efficacy of EGb: (1) the characteristics of the population studied and (2) the adequate selection of outcome measurements. In the EGb studies summarized in Tables 1 and 2, efficacy was systematically demonstrated when the population consisted of subjects cognitively impaired at baseline and rarely when it was a group of healthy volunteers ${ }^{1-7}$. Furthermore, the assessments measuring accuracy and speed, mostly related to working memory, were more successful for observing the EGb effect than the assessments measuring long term storage and retrieval abilities (delayed recall and recognition). Warot reported a striking positive effect of EGb on a memory test that assessed the immediate recall of 20 pictures $^{1}$. However, this difference was not due to an improvement in performance of the EGb group (mean recall of 15.5 pictures at baseline vs. 15.6 pictures 
Table 1 Memory and neuropsychological testing in healthy volunteers

\begin{tabular}{|c|c|c|}
\hline Publication (dosing regimen) & Mental domain or test & Outcome measure results \\
\hline Warot et al. ${ }^{1}(600 \mathrm{mg})$ & $\begin{array}{l}\text { Critical flicker frequency, choice reaction time } \\
\text { Sternberg number recognition (working memory), } \\
\text { picture recognition } \\
\text { Picture immediate recall }\end{array}$ & $\begin{array}{l}N^{*} \\
\text { NS } \\
\text { NS change from baseline in EGb group; } \\
\text { however, } P<0.05 \text { vs. placebo }\end{array}$ \\
\hline $\begin{array}{l}\text { Subhan and Hindmarch }{ }^{2} \\
(120,240,600 \mathrm{mg})\end{array}$ & $\begin{array}{l}\text { Critical flicker frequency, choice reaction time } \\
\text { Sternberg number recognition (working memory) }\end{array}$ & $\begin{array}{l}\text { Significant difference } 600 \mathrm{mg} \text { vs. } \\
\text { placebo for response time only }\end{array}$ \\
\hline
\end{tabular}

${ }^{*} \mathrm{NS}=$ No statistically significant difference from placebo.

1 h after $600 \mathrm{mg}$ EGb) but rather to a statistically significant decline in the performance of the placebo group (15.7 pictures at baseline vs. 13 at the $1 \mathrm{~h}$ session). The authors suggested that EGb may have a preventative rather than a memory enhancing effect. However, considering the healthy population enrolled in the study, the short duration of the trial and the lack of withdrawal phase, such preventative potential remains hypothetical.

The adequate dosage of EGb for achieving efficacy in these cognitive domains varied widely depending on the treatment regimen (single or chronic dosing). Overall, higher dosages (up to $600 \mathrm{mg} \mathrm{day}^{-1}$ ) seemed to be generally required for achieving efficacy on memory when acute single dose treatment was employed. The dose factor, however, has not been systematically handled in the currently available trials and additional studies comparing multiple dosages and different regimens are needed.

\section{Electrophysiology}

Electrophysiology is another field of interest in which to assess the effect of EGb on cognitive disorder, particularly because of the sensitivity of the electroencephalogram (EEG) to changes of vigilance and its use as a bioassay to assess the pharmacodynamic effects of CNS active agents. Theta/alpha ratio and profile of change in the beta, alpha, and theta bands have been considered the most adequate outcome measures for demonstrating the effects of the socalled 'nootropic' agents and 'cognitive activators'. These agents usually increase alpha and slow beta and/or theta band activity, resulting in an overall acceleration of the EEG background.

As shown in Table 3, EGb significantly affects the human EEG. In healthy volunteers, it has been shown that EGb induces an increase in the alpha and slow beta fractions of the EEG spectrum ${ }^{8-11}$. In patient populations (Table 4), it appears more likely to reduce the occurrence of slow activities (theta), thus decreasing the theta/alpha ratio ${ }^{12-15}$. Conflicting trial findings are mostly due to different baseline characteristics, circadian fluctuation of outcome measures, or complex dose-response curves. An example of interference between baseline characteristics and outcome measure can be seen in the Kunkel study, which failed to show a significant increase of alpha activity in healthy volunteers receiving a dose of $160 \mathrm{mg} \mathrm{day}^{-1} \mathrm{EGb}$ for 3 days 9 . Two of the inclusion criteria were a high alpha ratio in the baseline EEG (70\% of the background activity had to be in the alpha band) and a dominant alpha frequency between 9 and $11 \mathrm{~Hz}$. These extremely selective

Table 2 Memory and neuropsychological testing in an elderly population with mild to moderate cognitive impairment

\section{Publication}

(dosing regimen)

Allain et al. ${ }^{3} \quad$ Word/drawing immediate recall

$(320,600 \mathrm{mg})$

Grassel $^{4}$

(80 mg bid, 24 weeks)

Hofferberth $^{5}$

(40 mg tid, 8 weeks)

Israel et al. ${ }^{6}$

(80 mg bid, 12 weeks)

Wesnes et al. ${ }^{7}$ (40 mg tid, 12 weeks)
Dual coding time (speed of data processing)

Memory learning rate

Short term memory

Complex reaction time

Trail making (attention)

Learning rate and global memory

Attention and working memory

Mental fluidity (speed of data processing)

Benton/Word recall and recognition

Number matching, speed of processing

and mental efficiency
Outcome measure results

NS
Significant shift toward normal time in the EGb
groups
NS
Statistically significant difference from placebo at 24 weeks
Statistically significant difference from placebo at 8 weeks
NS
Statistically significant difference from placebo at 12 weeks
NS
Statistically significant difference from placebo at 8 and
12 weeks


Table 3 Electrophysiology in healthy volunteers

Publication

(dosing regimen)

Itil et al. ${ }^{8}$

$(40,120,240 \mathrm{mg}$ single dose $)$

Kunkel $^{9}$

(40, 80, $160 \mathrm{mg} 3$ days)

Le Bars et al. ${ }^{10}$

(80, 120, $240 \mathrm{mg}$ single dose)

Luhringer et al. ${ }^{11}$

(80, $160 \mathrm{mg}$ single and 5 days)
EEG changes

Increase alpha

Increase beta activity, decrease theta

Increase alpha, decrease theta

Increase alpha after single dose, decrease theta, increase beta after 5 days
Outcome measure results

Statistical significance from placebo,

all doses. Linear dose-response

Statistical significance from placebo after 3 days 80 and

$160 \mathrm{mg}$. No dose-response

Statistical significance from placebo $3 \mathrm{~h}$ after 120 or

$240 \mathrm{mg}$ (EEG and vigilance). Bimodal dose-response curve

Statistical significance from placebo $80,160 \mathrm{mg}$ single

dose and 5 days of chronic treatment criteria resulted in the exclusion of $50 \%$ of the healthy population screened. Not surprisingly, with such a rigid pre-selected EEG background, EGb induced only minor changes in the alpha band (no change in the occipital areas) but significant changes in the other frequencies. The EGb group showed an expected increase in slow beta activity but a surprising decrease in theta activity, which reached statistical significance compared with placebo.

An example of multiple interference between selection of study population, baseline EEG, and outcome measurement is found in the Gessner study, which enrolled a heterogeneous group of elderly volunteers suffering from non-specific cognitive decline ${ }^{14}$. Results based on mean comparisons of six EEG bands did not show significant changes after chronic dosing of $120 \mathrm{mg}$ day $^{-1}$ EGb during a 12 -week period. However, when a more homogeneous subgroup of patients with slow alpha background was selected (most impaired cases), EGb induced a significant decrease of the theta/alpha ratio and an increase of alpha dominant frequency, particularly during the resting EEG sessions. The authors attributed these changes to an improvement of vigilance. By selecting a subgroup of participants with the slowest alpha background, Gessner increased the probability of inclusion of subjects with more objective impairment and increased the magnitude of the treatment effect compared with placebo.

Discrepant results may also reflect a complex pharmacodynamic dose-response relationship. Kunkel failed to find a dose-response relationship for doses of 40, 80 and $160 \mathrm{mg} \mathrm{day}^{-1}$ after 3 days of chronic administration?. Itil, however, reported a statistically significant increase of alpha activity compared with placebo with a linear doseresponse relationship in a pilot study with multiple single doses of EGb $(40-240 \mathrm{mg})^{8}$. No statistically significant differences in the alpha band were observed when low (40 mg) and high (240 mg) dosages of EGb were compared. This lack of difference between EGb dosages compared with the discrimination from placebo suggests that part of the treatment effect was a correction of a decrease of alpha activity occurring with placebo. This hypothesis was further explored in the study of Le Bars ${ }^{10}$, which demonstrated a spontaneous decrease of alpha activity during the day of the placebo session, possibly due to well known circadian changes of EEG, apparently absent during the EGb sessions. Further, the dose response to EGb was found to follow a bimodal curve. In accordance with previous studies reporting a dose-response relationship, alpha changes that occurred immediately within $3 \mathrm{~h}$ after EGb intake were dose dependent and could be correlated to a decrease of drowsiness, as assessed by a vigilance selfrating scale. However, the late EEG changes observed at 7 and $9 \mathrm{~h}$ post-dosing were not dose dependent or correlated to significant changes on the vigilance rating scale. This finding is in accordance with the Kunkel study outcomes measuring the EGb effect after 3 days of treatment. This apparent double mode of action of EGb in the CNS will require further work to determine its clinical relevance. Nevertheless, it could obscure the interpretation of outcomes when effects of acute single doses are compared with those obtained after chronic treatments.

\section{Efficacy and safety in clinical studies}

A review of the literature by Kleijnen and Knipschild summarized the findings obtained before 1992 with EGb in patient populations suffering from cognitive impairment ${ }^{16}$. The studies, mainly undertaken in Europe, used as an inclusion criterion diagnosis of 'cerebral insufficiency'. This

Table 4 Electrophysiology in patient population with mild cognitive disorders

\section{Publication}

(dosing regimen)

Hofferberth $^{12}$

(80 mg tid, 12 weeks)

Rai et al. ${ }^{13}$

(40 mg tid, 24 weeks)

Gessner et al. ${ }^{14}$

(40 mg tid, 12 weeks)

Pidoux et al. ${ }^{15}$

(30 mg bid, 12 weeks)
EEG changes

Outcome measure results

Decrease theta/alpha ratio

Decrease slow activity

Decrease theta/alpha ratio

Decrease theta
Statistically significant difference from placebo at 12 weeks

Statistically significant difference from placebo at 12 weeks

NS. Statistically significant difference from placebo only in subgroup with slow baseline background Statistically significant difference from placebo at 12 weeks 
has no equivalent in the current classification of disease (ICD-10 or DSM-IV), but includes signs and symptoms very similar to those integrated in the so-called 'age associated memory impairment' syndrome, as well as in incipient dementia and mood-affective disorders. Of the 40 trials reviewed, 20 were double-blind studies and only eight met the minimum criteria to be considered an adequate Randomized Clinical Trial (RCT). All the studies used 100-160 mg day ${ }^{-1}$ EGb chronic doses and reported significant improvements of the clinical global impression after a minimum 6- to 12 -week treatment. The responder rate varied from 20 to $70 \%$ depending on the study. Results obtained in such a non-specific diagnosis framework, however, may not necessarily apply to pure cognitive disorders and are difficult to integrate in a review of EGb efficacy.

Another review of the literature by Letzel selected exclusively EGb studies enrolling patient populations with a diagnosis of cognitive disorder ${ }^{17}$. Of 25 randomized, double-blind, placebo-controlled EGb studies available at that time, eight explicitly included demented patients and three applied standard inclusion criteria of dementia using DSM-III-R. Overall, 23 studies reported a statistically significant difference favourable to EGb on at least one of the three levels of assessment: clinical global impression, psychometric, or social behaviour and daily living. Of the 18 studies that included simultaneous assessments of the clinical global impression and the psychometric, 10 demonstrated that EGb effect could be discriminated from placebo at a statistical level of significance. When only dementia was considered as the population of interest, seven of the eight trials available reported a significant treatment effect. Five of these trials showed positive results simultaneously in at least two levels of assessment (clinical global impression and psychometric). Regarding safety, no significant difference was found between EGb and placebo. In a study population of 739 patients, the most frequent events were gastrointestinal (19 patients, 2.6\%), headache (7 patients, 0.9\%), sleep disturbances and dizziness (3 patients, $0.4 \%$ ) and skin eruptions ( 2 patients, $0.3 \%)$.

Since Letzel's review, the efficacy of EGb has been further supported by results obtained from a multi-centre RCT conducted in the United States, which enrolled 327 patients with Alzheimer's Disease (AD) or Multi-Infarct Dementia $^{18}$. Whereas the largest study conducted in Europe used $240 \mathrm{mg} \mathrm{day}^{-1}$ EGb during a 24 week period and focused on dementia of mild to moderate severity ${ }^{19}$, the North American study tested a dose of $120 \mathrm{mg}^{\mathrm{day}}{ }^{-1}$ over 52 weeks and included patients with a broader range of impairment, as assessed by MMSE scores ranging from 9 to 26 and Global Deterioration Scale (GDS) scores of 3 to 6. At the 52-week endpoint, the group that received EGb did not show a significant worsening on the psychometric scale (cognitive subtest of the Alzheimer's Disease Assessment Scale or ADAS-Cog), whereas the placebo group showed a worsening of 1.5 points, resulting in a statistically significant difference favouring EGb (1.4 points, $P=0.04$ ). On the Geriatric Evaluation by Relative's Rating Instrument scale, used by the caregivers to assess daily living and social behaviour, mild improvement was observed for the EGb group, whereas the placebo group worsened. The mean treatment effect of 0.14 points was statistically significant $(P=0.004)$. No difference was found on the clinical global impression scale.

To facilitate a historical comparison between EGb

Table 5 Comparison of three randomized controlled trials with EGb in dementia with mild to moderate impairment

\section{Publication}

(dosing regimen)

Hofferberth $^{12}$

(21 AD patients on $240 \mathrm{mg} \mathrm{EGb}, 19$ patients

on placebo for 12 weeks)*

Kanowski et al. ${ }^{19}$

(79 patients on $240 \mathrm{mg} \mathrm{EGb}, 77$ patients on placebo for 24 weeks)

Le Bars et al. ${ }^{20}$

(154 patients on $120 \mathrm{mg} \mathrm{EGb}, 155$ patients on placebo: 26 weeks post hoc analysis)
Level of assessment

Outcome measure results

Complex reaction time

Favourable trend for EGb

Memory and attention with timed test (SKT)

Global impression, total score of the

Sandoz Clinical Assessment

Memory and attention with timed test (SKT)

Global impression (CGI)

Social behaviour (NAB)

2 out of 3 assessments

Memory, vigilance and complex performance (ADAS)

Global impression (CGI) Social behaviour (GERRI)
$P<0.001,50 \%$ responder rate by 5 SKT points with $\mathrm{EGb}, 0 \%$ with placebo

$76 \%$ responder rate

$P<0.05,38 \%$ responder rate at 4 points with EGb vs. $18 \%$ with placebo

$P<0.05,32 \%$ responder rate with EGb versus $17 \%$ with placebo

NS, $33 \%$ responder rate with EGb vs. $23 \%$ with placebo

$P<0.005,28 \%$ responder rate with $\mathrm{EGb}$ vs. $10 \%$ with placebo

$P<0.04,26 \%$ improvement 4 points with EGb vs. $17 \%$ with placebo

NS

$P<0.01,30 \%$ responder rate with EGb vs. $25 \%$ with placebo

${ }^{*} \mathrm{AD}$, Alzheimer's Disease. 
studies, a post hoc intent-to-treat analysis utilizing a 6month endpoint was recently performed on the North American data $\operatorname{set}^{20}$. A summary of the outcomes of this analysis is provided in Table 5, along with those from the trials of Hofferberth ${ }^{12}$ and Kanowski ${ }^{19}$ since they may be considered the most representative of the European data. Comparison of the North American results with those reported in the German studies might suggest that an increased EGb dose would result in an increased treatment effect. After a similar treatment duration (i.e. 24 weeks), the group in the Kanowski study treated with $240 \mathrm{mg}$ EGb showed a positive outcome on the CGI and there was a higher percentage of improved patients. In the EGb group, $38 \%$ of the patients reached the highest cut-off point on the cognitive scale vs. $27 \%$ in the North American study. Both showed a similar placebo response (18\%). The increase of the responder rate, however, does not seem to be linear with the increase in dosage. That is, doses higher than $240 \mathrm{mg}$ may not significantly improve the effects of treatment. This pattern of response was previously predicted by the EEG results of Le Bars ${ }^{10}$.

Ultimately, a control study with multiple doses of EGb will be needed to clarify the dose-response relationship and to help delineate the optimal regimen to obtain symptomatic improvement within at least a 6-month period. Future trials designed to assess changes in the course of the disease (i.e. preventative effect) would help delineate the role this special extract of Ginkgo biloba can play as an alternative to cholinesterase inhibitors or an adjunct agent for the treatment of dementia.

Overall, the efficacy of EGb has been demonstrated in cognitive disorders using neuropsychological assessments and electrophysiological tests. However, many trials have yielded discrepant findings, most likely due to differences in study population and trial design. To guarantee success in future research as well as in medical interventions, the population of interest should show an objective cognitive impairment at baseline, the outcome measures should include broad assessments of cognition (not exclusively memory tests) and treatment should explore doses higher than $240 \mathrm{mg} \mathrm{day}^{-1}$ in order to detect a plateau of response to EGb.

\section{References}

1 Warot D, Lacomblez L, Danjou P, Weiller E, Payan C, Puech AJ. Comparative effects of Ginkgo biloba extract on psychomotor performance and memory in healthy volunteers. (In French.) Therapie 1991; 46: 33-6.

2 Subhan Z, Hindmarch I. The psychopharmacological effects of Ginkgo biloba extract in normal healthy volunteers. Int.J. Clin. Pharm. Res. 1984; IV (2): 89-93.

3 Allain H, Raoul P, Lieury A, LeCoz F, Gandon JM, d'Arbigny P. Effect of two doses of Ginkgo biloba extract (EGb 761) on the dual-coding test in elderly subjects. Clin. Ther. 1993; 15: 54958.
4 Grassel E. The influence of Ginkgo biloba extract on mental performance, a double-blind study under computerized measurement conditions in patients with cerebral insufficiency. (In German.) Fortschr. Med. 1992; 110: 73-6.

5 Hofferberth B. Effect of Ginkgo biloba extract on neurophysiological and psychometric findings in patients with cerebroorganic syndrome. (In German.) Arzneim.-Forsch./Drug Res. 1989; 39 (II): 918-22.

6 Israel L, Dell'Accio E, Martin G, Hugonot R. Ginkgo biloba extract and memory training programs. Comparative assessment on elderly out-patients. (In French.) Psychol. Med. 1987; 19: 1431-9.

7 Wesnes K, Simmons D, Rook M, Simpson P. A double-blind placebo-controlled trial of tanakan in the treatment of idiopathic cognitive impairment in the elderly. Hum. Psychopharm. 1987; 2: 159-69.

8 Itil TM, Eralp E, Tsambis E, Itil KZ, Stein U. Central nervous system effects of Ginkgo biloba, a plant extract. Am. J. Ther. 1996; 3: 63-73.

9 Kunkel H. EEG profile of three different extractions of Ginkgo biloba. Neuropsychobiology 1993; 27: 40-5.

10 Le Bars P, Itil TM, Salzman C, Saito A, Bucci P. QEEG for dosefinding of new psychotropics and antidementia drugs In: Kimura J, Shibasaki H, eds. Recent Advances in Clinical Neurophysiology. Proceedings of the 10th International Congress of EMG and Clinical Neurophysiology, 15-19 October 1995, Kyoto, Japan. Amsterdam: Elsevier, 1996: 780-3.

11 Luthringer R, d'Arbigny P, Minot R, Toussaint M, Dago KT, Macher JP. EEG and EPs mapping profile of Ginkgo biloba extract (EGb 761). Clin. Neuropharm. 1992; 15 (Suppl. I): $152 \mathrm{~B}$.

12 Hofferberth B. The efficacy of EGb 761 in patients with senile dementia of the Alzheimer type, a double-blind, placebocontrolled study on different levels of investigation. Hum. Psychopharmacol. 1994; 9: 215-22.

13 Rai GS, Shovlin C, Wesnes KA. A double-blind, placebo controlled study of Ginkgo biloba extract ('Tanakan') in elderly out-patients with mild to moderate memory impairment. Curr. Med. Res. Opinion 1991; 12: 350-5.

14 Gessner B, Voelp A, Klasser M. Study of the long-term action of Ginkgo biloba extract on vigilance and mental performance as determined by means of quantitative pharmaco-EEG and psychometric measurements. Arzneim.-Forsch./Drug Res. 1985; 35: 1459-65.

15 Pidoux B, Bastien C, Niddam S. Clinical and quantitative EEG double-blind study of Ginkgo biloba extract (GBE). J. Cereb. Blood Flow Metab. 1983; 3 (Suppl. I): S556-7.

16 Kleijnen J, Knipschild P. Ginkgo biloba for cerebral insufficiency. Br. J. Clin. Pharmacol. 1992; 34: 352-8.

17 Letzel H, Haan J, Feil WB. Nootropics: Efficacy and tolerability of products from three active substance classes. J. Drug Dev. Clin. Pract. 1996; 8: 77-94.

18 Le Bars PL, Katz MM, Berman N, Itil TM, Freedman AM, Schatzberg AF. A placebo-controlled, double-blind, randomized trial of an extract of Ginkgo biloba for dementia. JAMA 1997; 278 (16): 1327-32.

19 Kanowski S, Herrmann WM, Stephan K, Wierich W, Horr R. Proof of efficacy of the Ginkgo biloba special extract EGb 761 in outpatients suffering from mild to moderate primary degenerative dementia of the Alzheimer type or multi-infarct dementia. Pharmacopsychiatry 1996; 29: 47-56.

20 Le Bars PL, Kieser M, Itil KZ. A 26-week analysis of a doubleblind, placebo-controlled trial of the Ginkgo biloba extract EGb 761 in dementia. Dement. Geriatr. Cogn. Disord. 2000; 11: $230-7$. 